\title{
El impacto psicológico de la pandemia de COVID-19 en el personal de salud: Un panorama preocupante
}

\author{
The psychological impact of the COVID-19 pandemic in the health care staff: \\ A worrisome landscape
}

\author{
Rodrigo A. Figueroa
}

Palabras clave: COVID-19; personal de salud; impacto psicológico.

La pandemia de COVID-19 ha impactado de manera dramática a la humanidad en casi todas las formas imaginables. A la fecha de la redacción de esta editorial, el SARS-CoV-2 ha cobrado la vida de casi un millón de personas, y ha contagiado a más de 30 millones (Roser et al., 2020). La mayor parte de los países del mundo ha restringido la movilidad de sus ciudadanos, con algunas excepciones como Corea del Sur (Campbell, (2000), Japón (The associated press, 2020), Suecia ( Birchall, 2020), Uruguay ( AFP \& Scarpinelli, 2020) y algunos estados de Norte América ( Mervosh, 2020) y Brasil (Araújo, 2020).

El ámbito educacional ha sido también fuertemente impactado. Así, al igual que en nuestra facultad, más del $90 \%$ de los estudiantes del mundo han debido suspender sus clases presenciales (UNESCO, 2020), lo que ha precipitado una verdadera, pero también desigual revolución en la educación a distancia ( Nature, 2020).

En la economía, el mundo se encuentra actualmente en una recesión que podría llegar a ser la peor que haya enfrentado el planeta desde la segunda guerra mundial (World Bank, 2020), con un 13,1\% de cesantía en Chile (INE, 2020). Sólo en E.E.U.U. se ha estimado que "la desesperación" asociada al desempleo y aislamiento social podría producir 75000 muertes adicionales por suicidio o abuso de alcohol y drogas (Petterson, 2020).

Un grupo particularmente afectado por la pandemia es el de los profesionales y funcionarios de la salud. Se trata de un grupo que ha estado especialmente exigido en esta pandemia, tanto en lo laboral como en lo personal. Varios han estado expuestos a sobrecarga asistencial y a un mayor riesgo de contagio para sí mismos o sus familias. En efecto, al 18 de agosto de este año, el sitio de contenidos médicos Medscape tenía recopilados los nombres de 2012 funcionarios de la salud del mundo fallecidos por la pandemia (Frellick, 2020).
No todos los funcionarios de la salud han tenidoel mismo nivel de demanda durante esta emergencia. Algunos han tenido que redoblar turnos o ir en apoyo de sus colegas, mientras que otros se han visto afectados por una importante disminución de actividad laboral. Varios han tenido que asumir roles para los cuales no estaban capacitados, o han tenido que reentrenarse de manera acelerada para recuperar conocimientos ya perdidos hace años. Esta situación ha expuesto a los funcionarios a lo que se ha dado a llamar"daño moral" (Greenberg et al., 2020), una condición que afecta a las personas cuando en virtud de su rol profesional deben hacer cosas que transgredan sus principios, en este caso prestar atención clínica sin la convicción de tener las competencias para hacerlo de manera óptima. Otros profesionales, en especial mujeres, han debido lidiar con la "doble presencia", que corresponde a la condición laboral en la que el trabajador se ve obligado a tener que prestar simultáneamente atención a problemas del trabajo y domésticos. Esta condición, habitual en trabajadores que hacen turnos, ha cobrado aún más relevancia durante esta pandemia, con niños en casa que requieren supervisión permanente y una red social de apoyo limitada por el confinamiento. Algunos trabajadores de la salud, especialmente al principio de la pandemia, fueron objeto de exclusióny/o rechazo en sus comunidades, donde fueron obligados a usar accesos diferenciados, o a trasladar su vivienda. La exposición a escenas de muerte de pacientes sin la compañía de sus familiares producto de las restricciones de visita es otra más de las experiencias altamente estresantes a las que ha estado sometido este grupo, lo que en conjunto determina la presencia de varios factores de riesgo psicosocial en el trabajo (Ministerio de Salud de Chile, 2013).

En efecto, estudios internacionales han mostrado una alta prevalencia de síntomas psicológicos en trabajadores de la salud abocados al trabajo en epidemias. Por ejemplo, en un metaanálisis de 115 artículos publicados hasta abril de 2020, que incluyó a 60458 trabajadores de la salud involucrados en el enfrentamiento de

(1) Departamento de Psiquiatría, Escuela de Medicina, Pontificia Universidad Católica de Chile

Autor de correspondencia: rfiguerc@uc.cl 
SARS/MERS/COVID-19, un 62,5\% de ellos reportó "preocupaciones generales de salud", 43,7\% miedo, 37,9\% insomnio, 37,8\% malestar psicológico, 34,4\% burnout, 29,0\% cuadros ansiosos, 26,3\% síntomas depresivos, 20,7\% fenómenos típicos del trastorno de estrés post-traumático, $16,1 \%$ somatización, y 14,0\% sentimientos de ser objeto de estigmatización (Salazar de Pablo et al., 2020). Por otra parte, en un estudio transversal de 1257 trabajadores de la salud de China abocados al cuidado de pacientes COVID-19 en 34 hospitales, un 50,4\% reportó síntomas depresivos, 44,6\% síntomas de ansiedad, 34,0\% insomnio, y 71,5\% malestar psicológico (Lai et al., 2020). En otro metaanálisis de 14 estudios se reportó que un $2,2-14,5 \%$ de los funcionarios de salud reportaron síntomas "severos" (Bohlken et al., 2020). Entre los factores de riesgo para un mayor impacto psicológico se han reportado: el tener una menor experiencia laboral, trabajo a jornada parcial, ser enfermera en comparación con médicos, insuficiente capacitación, percepción de falta de apoyo organizacional, compensaciones insuficientes, percibir estigmatización, bajo nivel socioeconómico, mayor exposición a noticias sobre la pandemia, sexo femenino, menor edad, madres/padres de hijos dependientes, y temor a contagiar a familiares (Kisely et al., 2020; Luo et al., 2020; Pappa et al., 2020).

En Chile, al igual que en el resto del mundo, la situación de nuestros trabajadores de la salud también es preocupante. El "Informe preliminar № 1 Chile - Síntomas depresivos y malestar psicológico", (Universidad de Chile, 2020) desarrollado por académicos de varias universidades chilenas en asociación con investigadores de 30 países, mostró que, en una muestra de 954 trabajadores de la salud de todo el país, un 31,4\% reportó síntomas depresivos moderados o graves, casi el doble que lo observado en otro estudio en Chile previo a la pandemia. De especial relevancia, un 7,1\% de los trabajadores encuestados reportó haber tenido ideas suicidas. Por otra parte, un 54,8\% de quienes respondieron podrían tener un trastorno mental común (i.e. ansioso, depresivo, adaptativo), una cifra más de tres veces superior a la encontrada en estudios de prevalencia previos en nuestro país (Universidad de Chile,2020).

Un grupo especial de actores que ha sufrido una importante disrupción en sus rutinas ha sido el de los alumnos de medicina de los últimos años de la carrera (internos). En este número de Ars Médica un grupo de ellos relata su experiencia participando en actividades clínicas ligadas a la pandemia (Ballart et al., 2020), dejando testimonio de cómo han debido enfrentar la sobrecarga asistencial, la incertidumbre, la soledad, y la muerte de sus pacientes, y cómo - finalmente - esas experiencias se han configurado como hitos en su formación, no sólo en la dimensión técnica de la medicina, sino en la humana. Los invito a leer su testimonio con atención y felicito a los autores por su coraje y por la decisión de haber compartido su experiencia en esta revista.
Para finalizar, u nas p alabras d e c autela. E s i mportante tener p resente que los estudios de prevalencia de síntomas señalados en esta editorial son estudios de auto reporte, y por lo tanto tienen las limitaciones propias de este tipo de instrumentos. Los instrumentos de auto reporte, si bien ampliamente utilizados en estudios de salud mental, y de enorme utilidad cuando están bien validados, pueden tener importantes sesgos producto de deseabilidad social o fallas de memoria (Althubaiti, 2016). También pueden tener sesgos por una baja capacidad de introspección, una falta de comprensión sobre lo que se pregunta, o sesgos cognitivos que favorezcan respuestas extremas o dicotómicas en fenómenos de naturaleza continua, entre otros (Austin et al., 1998; Balakrishnan, 1999; Fan et al., 2006). Es importante tener presente estas limitaciones, para no sacar conclusiones apresuradas respecto al impacto de esta epidemia sobre la incidencia y prevalencia de trastornos psiquiátricos formales. Dado que existe evidencia de que la correlación entre el auto reporte y la entrevista clínica puede ser en algunos casos pobre (Eaton et al., 2000), se hace necesario complementar las investigaciones existentes con estudios que utilicen entrevistas clínicas estructuradas, permitiendo de este modo incorporar la profundidad y precisión que da el examen mental en el diagnóstico de los trastornos psiquiátricos.

\section{Referencias}

AFP \& Scarpinelli L. (2020) Uruguay rides out COVID threat without imposing a lockdown. Manila Standard. Accedido en https://manilastandard.net/news/top-stories/327561/uruguay-rides-out-covid-threat-without-imposing-a-lockdown.html el 27 de septiembre de 2020

Althubaiti A. (2016). Information bias in health research: definition, pitfalls, and adjustment methods. Journal of Multidisciplinary Healthcare 9, 211-217.

Austin EJ, Deary IJ, Gibson GJ, McGregor MJ. \& Dent JB. (1998). Individual response spread in self-report scales: personality correlations and consequences. Personality and Individual Differences 24, 421-438.

Araújo F. (2020) Justiça nega pedido do MP para obrigar governo e prefeitura de Boa Vista a decretar "lockdown" Roraima G1. Accedido en https://g1.globo.com/rr/roraima/noticia/2020/06/03/justica-nega-pedido-do-mp-para-obrigar-governo-e-prefeitura-de-boa-vista-a-decretar-lockdown.ghtml el 27 de septiembre de 2020.

Balakrishnan JD. (1999). Decision processes in discrimination: Fundamental misrepresentations of signal detection theory. Journal of Experimental Psychology: Human Perception and Performance 25, 1189-1206.

Ballart M, González J, Larraín S, Muñoz F, Pérez S. \& Andresen M. (2020) Experiencia del internado de medicina durante la pandemia por Covid-19. Ars Medica, Revista de Ciencias Médicas 45, 72-75. 
Birchall G. (2020) Sweden says no to quarantine - is this the most reckless or the most proportionate Covid-19 response in the West?. RTWorld News. Accedido en https://www.rt.com/news/484515-sweden-covid-no-lockdown/ el 27 de septiembre de 2020.

Bohlken J, Schömig F, Lemke MR, Pumberger M. \& Riedel-Heller SG. (2020). [COVID-19 Pandemic: Stress Experience of Healthcare Workers - A Short Current Review]. Psychiatrische Praxis 47, 190-197.

Campbell C. (2000) South Korea's Health Minister on How His Country Is Beating Coronavirus Without a Lockdown Time. Accedido en https://time.com/5830594/south-korea-covid19-coronavirus/ el 27 de septiembre de 2020 .

Eaton WW, Neufeld K, Chen LS. \& Cai G. (2000). A comparison of self-report and clinical diagnostic interviews for depression: diagnostic interview schedule and schedules for clinical assessment in neuropsychiatry in the Baltimore epidemiologic catchment area follow-up. Archives of General Psychiatry 57, 217-222.

Fan X, Miller BC, Park K.-E, Winward BW, Christensen M, Grotevant HD. \&Tai RH. (2006). An Exploratory Study about Inaccuracy and Invalidity in Adolescent Self-Report Surveys. Field Methods 18, 223-244.

Frellick M. (2020) Remembering Thousands of World's Healthcare Workers Lost to COVID. Accedido en https://www.medscape.com/ viewarticle/936117 el 27 de septiembre de 2020.

Greenberg N, Docherty M, Gnanapragasam S. \& Wessely S. (2020). Managing mental health challenges faced by healthcare workers during covid-19 pandemic. BMJ (Clinical Research Ed.) 368, m1211.

INE (2020). INE publica resultados de la Encuesta Nacional de Empleo del trimestre mayo-julio de 2020. Accedido en https:// www.ine.cl/prensa/2020/08/28/ine-publica-resultados-de-la-encuesta-nacional-de-empleo-del-trimestre-mayo-julio-de-2020 el 27 de septiembre de 2020 .

Kisely S, Warren N, McMahon L, Dalais C, Henry I. \& Siskind, D. (2020). Occurrence, prevention, and management of the psychological effects of emerging virus outbreaks on healthcare workers: rapid review and meta-analysis. BMJ (Clinical Research Ed.) 369, m1642.

Lai J, Ma S, Wang Y, Cai Z, Hu J, Wei N. et al. (2020). Factors associated with mental health outcomes among health care workers exposed to coronavirus disease 2019. JAMA Network Open 3, e203976.

Luo M, Guo L, Yu M, Jiang W \& Wang H. (2020). The psychological and mental impact of coronavirus disease 2019 (COVID-19) on medical staff and general public - A systematic review and meta-analysis. Psychiatry Research 291, 113190.
Mervosh S, Lu D. \& Vanessa Swales (2020) See Which States and Cities Have Told Residents to Stay at Home - The New York Times. Accedido en https://www.nytimes.com/interactive/2020/us/coronavirus-stay-at-home-order.html el 27 de septiembre de 2020.

Ministerio de Salud de Chile. Protocolo de vigilancia de riesgos psicosociales en el trabajo (2013). Accedido en https://www.minsal. $\mathrm{cl} /$ portal/url/item/e039772356757886e040010165014a72.pdf el 27 de septiembre de 2020 .

Nature (2020) Online learning cannot just be for those who can afford its technology. (2020). Nature 585, 482 Accedido en https://www. nature.com/articles/d41586-020-02709-3 el 27 de septiembre 2020

Pappa S, Ntella V, Giannakas T, Giannakoulis VG, Papoutsi E, \& Katsaounou P. (2020). Prevalence of depression, anxiety, and insomnia among healthcare workers during the COVID-19 pandemic: A systematic review and meta-analysis. Brain, Behavior, and Immunity 88, 901-907.

Petterson S, Westfall JM. \&Miller BF. (2020). Projected Deaths of Despair During COVID-19.Well Being Trust. Accedido en https://wellbeingtrust. org/areas-of-focus/policy-and-advocacy/reports/projected-deaths-of-despair-during-covid-19/ el 27 de septiembre de 2020.

Roser M, Ritchie H, Ortiz-Ospina E. \& Hasell J. (2020). Coronavirus Pandemic (COVID-19) - Statistics and Research. Our World in Data.

Salazar de Pablo G, Vaquerizo-Serrano J, Catalan A, Arango C, Moreno C, Ferre F. et al. (2020). Impact of coronavirus syndromes on physical and mental health of health care workers: Systematic review and meta-analysis. Journal of Affective Disorders 275, 48-57.

The associated press (2020) Japan's state of emergency is no lockdown. What's in it?. The Asahi Shimbun. Accedido en http://www. asahi.com/ajw/articles/13281427 el 27 de septiembre de 2020.

Universidad de Chile. (2020) Primer informe The COVID-19 Health Care Workers Study - Escuela de Salud Pública - Accedido en http:// www.saludpublica.uchile.cl/noticias/167313/primer-informe-thecovid-19-health-care-workers-study el 27 de septiembre de 2020.

UNESCO (2020). School closures caused by Coronavirus (Covid-19). Accedido en https://en.unesco.org/covid19/educationresponse el 27 de septiembre de 2020 .

World Bank (2020) COVID-19 to Plunge Global Economy into Worst Recession since. Accedido en https://www.worldbank. org/en/news/press-release/2020/06/08/covid-19-to-plungeglobal-economy-into-worst-recession-since-world-war-ii el 27 de septiembre de 2020 